\title{
Regional parent flood frequency distributions in Europe - Part 2: Climate and scale controls
}

\author{
J. L. Salinas ${ }^{1}$, A. Castellarin ${ }^{2}$, S. Kohnová ${ }^{3}$, and T. R. Kjeldsen ${ }^{4}$ \\ ${ }^{1}$ Institute of Hydraulic Engineering and Water Resources Management, Vienna University of Technology, Vienna, Austria \\ ${ }^{2}$ Department DICAM, School of Civil Engineering, University of Bologna, Bologna, Italy \\ ${ }^{3}$ Department of Land and Water Resources Management, Faculty of Civil Engineering, \\ SUT Bratislava, Slovak Republic \\ ${ }^{4}$ Department of Architecture and Civil Engineering, University of Bath, Bath, UK
}

Correspondence to: J. L. Salinas (salinas@hydro.tuwien.ac.at)

Received: 2 May 2013 - Published in Hydrol. Earth Syst. Sci. Discuss.: 22 May 2013

Revised: 11 September 2014 - Accepted: 15 September 2014 - Published: 5 November 2014

\begin{abstract}
This study aims to better understand the effect of catchment scale and climate on the statistical properties of regional flood frequency distributions. A database of Lmoment ratios of annual maximum series (AMS) of peak discharges from Austria, Italy and Slovakia, involving a total of 813 catchments with more than $25 \mathrm{yr}$ of record length is presented, together with mean annual precipitation (MAP) and basin area as catchment descriptors surrogates of climate and scale controls. A purely data-based investigation performed on the database shows that the generalized extreme value (GEV) distribution provides a better representation of the averaged sample L-moment ratios compared to the other distributions considered, for catchments with medium to higher values of MAP independently of catchment area, while the three-parameter lognormal distribution is probably a more appropriate choice for drier (lower MAP) intermediate-sized catchments, which presented higher skewness values. Sample L-moment ratios do not follow systematically any of the theoretical two-parameter distributions. In particular, the averaged values of L-coefficient of skewness (L-Cs) are always larger than Gumbel's fixed L-Cs. The results presented in this paper contribute to the progress in defining a set of "process-driven" pan-European flood frequency distributions and to assess possible effects of environmental change on its properties.
\end{abstract}

\section{Introduction}

The companion paper by Salinas et al. (2014) presents a newly established database of flood L-moments from $13 \mathrm{Eu}$ ropean countries. Based on a preliminary visual inspection and some basic averaging, the generalized extreme value (GEV) distribution appeared to be a potential pan-European flood frequency distribution. However, Monte Carlo simulations showed that there is not enough statistical evidence for the existence of a single three-parameter distribution suitable for representing flood frequency regimes all over Europe. This supports the fact of statistical model selection being a topical issue in hydrology and flood frequency analysis in particular (see e.g. Laio et al., 2009). Literature shows recent advances on how to combine different theoretical models together to improve the representation of the local flood frequency regime through multimodel approaches when the reproduction provided by a single theoretical model is not satisfactory (Bogdanowicz, 2010; Kochanek et al., 2012).

Concerning probabilistic model selection, Hosking and Wallis (1997), Vogel and Fennessey (1993) and Peel et al. (2001), among others, recommend using the L-moment ratio diagrams to guide the selection of the most suitable parent flood frequency distribution. L-moment ratio diagrams have been used for detecting suitable parents for a wide spectrum of geohydrological extremes (precipitation depths, flood flows, earthquake magnitudes and others) observed in different geographical contexts around the world (see e.g. Vogel and Wilson, 1996; Robson and Reed, 1999; Thompson et al., 2007). In the hydrological application of L-moment 
ratio diagrams, and referring more specifically to flood frequency analysis, usage of these kind of diagrams requires sample statistics, which are unavailable or highly uncertain for ungauged or poorly gauged regions. This is one of the reasons why many authors have performed data-based analyses trying to find relationships between sample moments of the flood series and catchment descriptors. Among many others, Schaefer (1990), Farquharson et al. (1992), Meigh et al. (1997), Blöschl and Sivapalan (1997), Iacobellis et al. (2002), Brath et al. (2003), Merz and Blöschl (2003), Di Baldassarre et al. (2006), Merz and Blöschl (2009), Padi et al. (2011), and Viglione et al. (2012) have found regional relationships between sample moments (mainly mean annual flood and coefficient of variation) and catchment area, mean annual rainfall, and other lumped climatic indicators such as aridity. While this correlation may change due to local processes, there is more or less a consensus in the literature that the mean annual specific flood and coefficient of variation of peak annual discharges increase with decreasing catchment size, as well as with decreasing mean annual precipitation (MAP), although this last effect is more clear in arid climates. The current paper tries to go a step further and relate the catchment descriptors to the probabilistic model selection through their controls on the flood moments.

On an applied level, existing guidelines give recommendations on which statistical model, i.e. regional or local parent distribution, to use. This choice could have an important effect on the estimation of high return period flood quantiles due to the different behaviour of the tails of the distribution functions (see e.g. El Adlouni et al., 2008). In some occasions, these recommendations are not justified by any evidence from the local data, or are simply inspired or adapted from analogue guidelines in other countries. Keeping in mind the need for a more effective use of existing data, a key scientific and practical challenge for improved risk assessment is a pan-European comparison and evaluation of the consistency of estimates across methods, physiographic regions and a variety of spatial scales in order to ensure comparable flood frequency estimates and safety measures across Europe, as requested by the Directive 2007/60/EC. In fact, it is of utmost importance for the implementation of the Flood Directive that state-of-the-art and harmonized methods are used to estimate extreme flood frequencies to obtain consistent values for locations where rivers cross national borders.

On the basis of these considerations, this paper addresses the two main scientific questions: can we quantify the main physical controls on the shape of the flood frequency distribution at a regional scale? Can we represent these controls graphically on L-moment ratio diagrams to guide the selection of suitable parent distribution on the basis of fundamental catchment descriptors? For this purpose, a subset of the data presented in Salinas et al. (2014) with a total of 1132 catchments from Austria, Italy, and Slovakia is used to study the control of commonly available physiographic and climatic characteristics (catchment size and mean annual precipitation) on the properties of the underlying probability distribution of flood flow.

\section{Description of the data set}

The analysis presented in this paper focuses on annual maximum series (AMS) of peak flow from three national databases, namely Austrian, Italian and Slovakian, and it addresses the control of catchment size and climate, respectively, on the flood frequency regime. In particular, the analysis considers catchment area and MAP as catchment descriptors for the three data sets. The flood data was shared in the frame of the FloodFreq COST Action ES0901 (Kjeldsen, 2011; Castellarin et al., 2012) and constitutes a subset of the database presented in Salinas et al. (2014). The countries selected for this study were able to share not only the discharge data but the two catchment descriptors mentioned as well, while the rest of the countries had some kind of limitation in this sense.

On the choice of catchment area and mean annual precipitation, previous studies have proven them to exert significant control on the frequency regime of hydrological extremes (see e.g. Schaefer, 1990; Blöschl and Sivapalan, 1997; Brath et al., 2003; Di Baldassarre et al., 2006; Padi et al., 2011). They can be regarded as lumped catchment descriptors used as surrogate covariates representing the spatially distributed and complex hydrological processes controlling the catchment flood response. Precisely, the area of the basin is an indicator of the scale interplays between catchment processes and rainfall (Blöschl and Sivapalan, 1995), while mean annual precipitation acts as control of probabilistic behaviour of floods through its effect on antecedent soil moisture conditions (Sivapalan et al., 2005), and also provides an indication about other local and atmospheric process.

When combined, the three national data sets consist of AMS from a total of 1132 catchments (Austria, 676 gauges; Italy, 282 gauges; and Slovakia, 174 gauges). Table 1 describes the data set in terms of catchment area, MAP, record length of annual maximum series ( $n$ ), sample L-coefficient of variation (L-Cv), L-coefficient of skewness (L-Cs) and L-coefficient of kurtosis (L-Ck) for the case study. The geographical locations of the considered stream gauges are shown on the map in Fig. 1. From a purely visual analysis, due to the lack of detailed information on the hydrological regimes, the database is dominated by catchments with humid and continental climates, with a big proportion of mountainous catchments (where snow is supposed to play a significant role, but could not be included in the analysis due to the lack of this kind of data), and only a small percentage of catchments that could be considered Mediterranean or arid located in Italy.

As illustrated in the scatterplot of Fig. 2, the data set includes a range of values for catchment area and MAP, and does not show any statistically significant correlation 
Table 1. Summary of the Austrian, Italian and Slovakian national data sets. Information on the distribution of catchment area, MAP, record length $(n)$ and sample L-moment ratios of the annual flood sequences is given.

\begin{tabular}{lrrrrrr}
\hline & $\begin{array}{r}\text { Area } \\
\left(\mathrm{km}^{2}\right)\end{array}$ & $\begin{array}{r}\text { MAP } \\
\left(\mathrm{mm} \mathrm{yr}^{-1}\right)\end{array}$ & $\begin{array}{r}n \\
(\mathrm{yr})\end{array}$ & $\begin{array}{r}\mathrm{L}-\mathrm{Cv} \\
(-)\end{array}$ & $\begin{array}{r}\text { L-Cs } \\
(-)\end{array}$ & $\begin{array}{r}\text { L-Ck } \\
(-)\end{array}$ \\
\hline Min. & 4.6 & 501.7 & 9 & 0.0152 & -0.1209 & -0.1583 \\
1st quartile & 64.9 & 902.8 & 22 & 0.2194 & 0.1777 & 0.1268 \\
Median & 157.0 & 1112.0 & 34 & 0.2763 & 0.2705 & 0.1905 \\
Mean & 2096.5 & 1163.6 & 38 & 0.2945 & 0.2782 & 0.2074 \\
3rd quartile & 534.0 & 1369.3 & 47 & 0.3558 & 0.3733 & 0.2730 \\
Max. & 131488.0 & 2312.3 & 182 & 0.7691 & 0.7737 & 0.7132 \\
\hline
\end{tabular}

between the two (i.e. sample Pearson coefficient is equal to -0.010 , and the null hypothesis of zero correlation is associated with a $p$ value of 0.732 ). It is worth noting here that, as illustrated in Fig. 2, very large catchments (catchment areas larger than $10000 \mathrm{~km}^{2}$ ) are associated with medium MAP values (about $1200 \mathrm{~mm} \mathrm{yr}^{-1}$ ). Therefore, very large catchments in the study area are neither "wetter" nor "drier" catchments ("wetter" and "drier" as defined in Sect. 3.1), and this is an important element for the analyses described in Sects. 3.1 and 3.2.

As in the companion paper by Salinas et al. (2014), the framework used to analyse the flood data is the L-moment environment, and, in particular, through the use of L-moment ratio diagrams. Both L-Cs-L-Cv and L-Cs-L-Ck diagrams are used, as several two- and three-parameter candidate distributions are investigated.

\section{Results}

\subsection{Area and MAP control on sample L-moments}

A detailed exploration was undertaken to better understand the controls on the flood frequency regime exerted by physiographic and climatological factors, represented here by area and MAP. To minimize the possible effects of sampling variability associated with short records when estimating higher-order sample L-moments (see e.g. Viglione, 2010), the minimum record length was set to $25 \mathrm{yr}$ of data, reducing the data set to a total of 813 catchments (Austria, 493 gauges; Italy, 151 gauges; and Slovakia, 169 gauges). The combined data set was divided into six smaller subsets based on thresholds defined as the 20 and $80 \%$ quantiles of the catchment descriptor values. For convenience, the following subsets are defined to characterize the catchments according to size: smaller catchments $\left(\right.$ area $\left.<55 \mathrm{~km}^{2}\right)$, intermediate catchments $\left(55 \mathrm{~km}^{2}<\right.$ area $\left.<730 \mathrm{~km}^{2}\right)$ and larger catchments (area $>730 \mathrm{~km}^{2}$ ). Analogously, the catchments were classified based on MAP as drier catchments (MAP $<860 \mathrm{~mm} \mathrm{yr}^{-1}$ ), medium catchments $\left(860 \mathrm{~mm} \mathrm{yr}^{-1}<\mathrm{MAP}<1420 \mathrm{~mm} \mathrm{yr}^{-1}\right)$ and wetter catchments $\left(\mathrm{MAP}>1420 \mathrm{~mm} \mathrm{yr}^{-1}\right.$ ) (see also Fig. 2). The adjectives drier and wetter, and smaller and larger are relative to the distribution of wetness and sizes of the study data set. The 20 and $80 \%$ quantiles were selected after a set of preliminary trials as they enabled us to enhance the representation of the peculiarities in the flood frequency regimes for drier against wetter and for larger against smaller catchments for the considered data set.

For each of the wetness and size subsets, the record length weighted moving average (WMA) values of samples L-Cv, L-Cs and L-Ck were computed using a window of 70 catchments, where the 70 neighbouring catchments were selected by taking the closest catchments in terms of the considered descriptor (area or MAP). For each sample of the 70 catchments, the associated WMA value was plotted against the corresponding mean of catchment descriptor (area or MAP) as shown in Fig. 3 for each of the six subsets. Note that in this context, each individual WMA value has a regional validity, as it is derived from a pooling group of 70 sites, defined based on the similarity in terms of catchment size and rainfall regime (MAP). For example, the first point of the yellow line in Fig. 3a represents a non-contiguous region with MAP $<860 \mathrm{~mm} \mathrm{yr}^{-1}$ (drier catchments) and the 70 smallest sizes of the subset (in this case, catchment areas from 36 to $103 \mathrm{~km}^{2}$ ). The minimum amount of information for each point, assuming serial and spatial independence of the stations, would correspond to $70 \times 25=1750$ stationyears of data. The width of the window (i.e. 70 sites) provides a trade-off between the desire to effectively identify and visualize larger-scale structures in the data set and local deviations from the averaging process, and the conflicting need to work on larger samples to reduce the effects of sampling uncertainty.

Considering the WMA values plotted in Fig. 3, an observable feature is a general tendency for all the L-moment ratios to decrease with increasing area and MAP values. This is a result already reported in the literature for the case of conventional product moments, with special focus on scale effect on the coefficient of variation of the flood distribution (see e.g. Schaefer, 1990; Blöschl and Sivapalan, 1997; Brath et al., 2003; Merz and Blöschl, 2003; Di Baldassarre et al., 2006; Merz and Blöschl, 2009; Padi et al., 2011; Viglione 


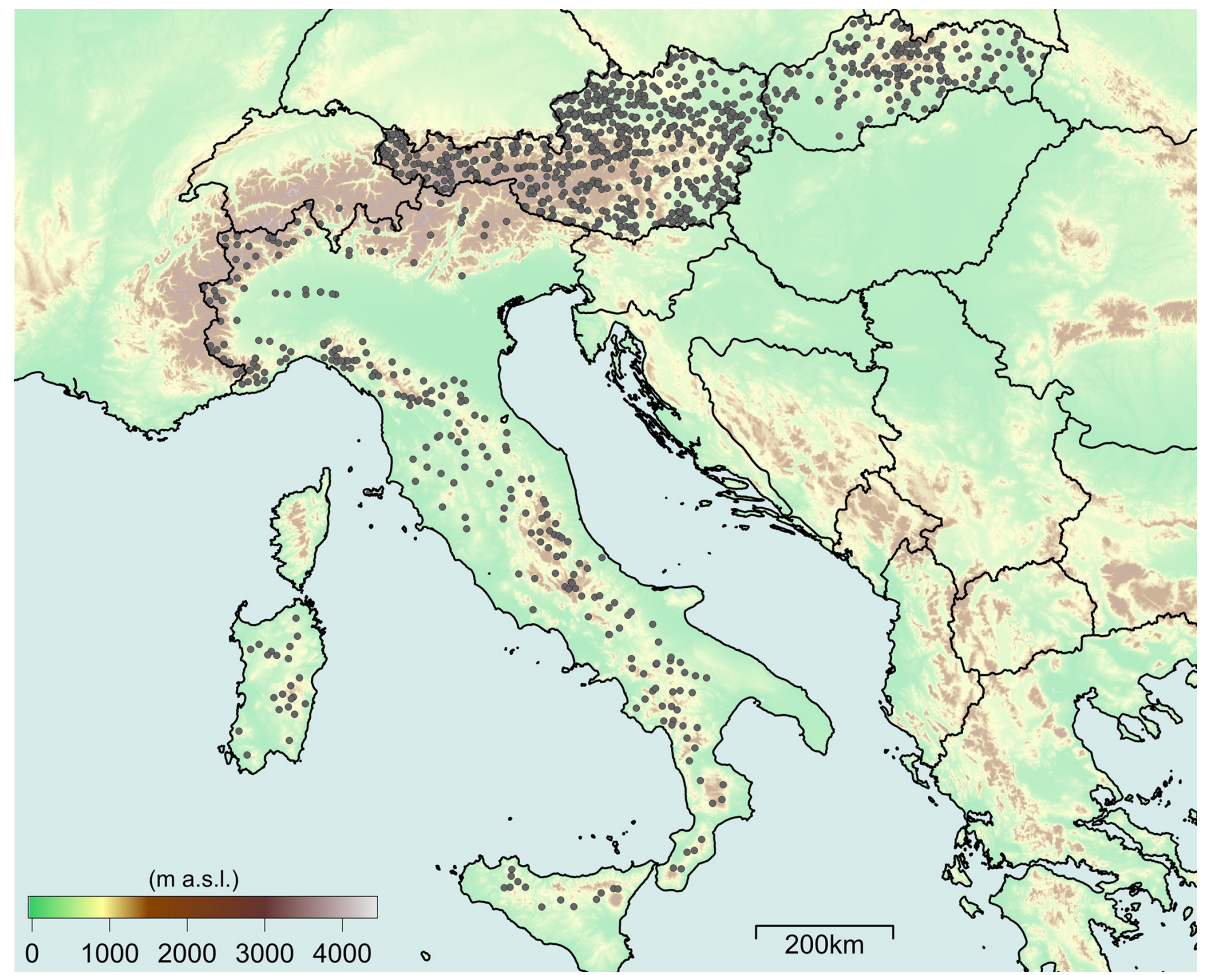

Figure 1. Map showing the location of the 1132 considered Austrian, Italian and Slovakian gauging stations (points). Colour scale in the background represents terrain elevation in metres a.s.l. (above sea level).

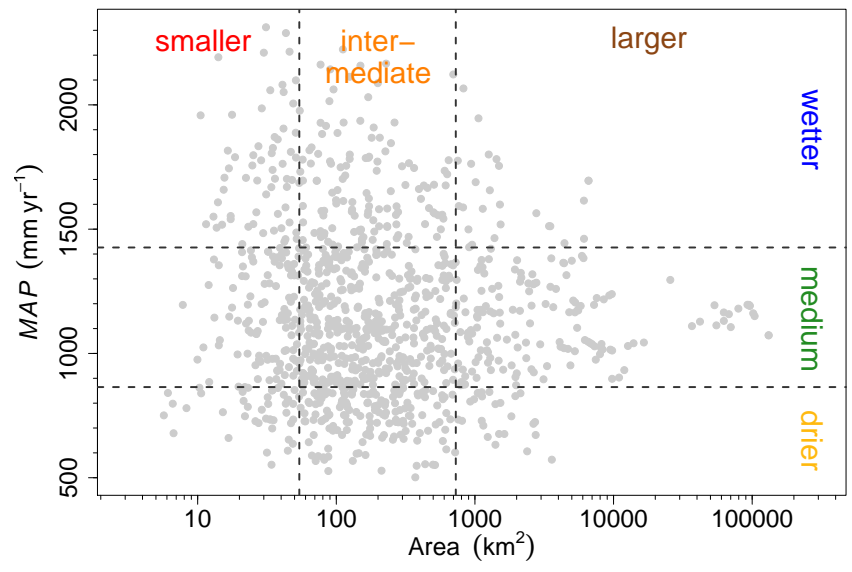

Figure 2. Catchment characteristics of the Austrian, Slovakian and Italian data sets. For each catchment, MAP is plotted against catchment area (grey circles). Black, dashed lines represent the 20 and $80 \%$ quantiles for each catchment descriptor, defining the subsets as smaller (area $<55 \mathrm{~km}^{2}$ ), intermediate $\left(55 \mathrm{~km}^{2}<\right.$ area $<730 \mathrm{~km}^{2}$ ), larger $\left(\operatorname{area}>730 \mathrm{~km}^{2}\right)$, drier $\left(\mathrm{MAP}<860 \mathrm{~mm} \mathrm{yr}^{-1}\right)$, medium $\left(860 \mathrm{~mm} \mathrm{yr}^{-1}<\mathrm{MAP}<1420 \mathrm{~mm} \mathrm{yr}^{-1}\right)$, and wetter catchments $\left(\mathrm{MAP}>1420 \mathrm{~mm} \mathrm{yr}^{-1}\right)$. et al., 2012). Farquharson et al. (1992) and Meigh et al. (1997) also found an increase of coefficient of variation and skewness with increasing aridity, which is consistent with this study, if one takes the lower MAP values as an indicator for a higher aridity. The largest gradients in Fig. 3 are observed for L-Cv, followed by L-Cs and, finally, L-Ck, confirming the lower variability of higher-order L-moments in space (see e.g. Hosking and Wallis, 1997), and also showing that the lower-order L-moment ratios have a stronger link to catchment and climate properties than higher-order Lmoment ratios. It is also noticeable that the WMA lines are not evenly spaced, which indicates a degree of non-linearity between the flood characteristics and the catchment properties. This is particularly evident when considering L-Cv plotted against both catchment area and MAP in Figs. 3a and b, for L-Cs plotted against MAP (subsets defined based on area) in Fig. 3d and, to some extent, for L-Ck plotted against MAP (again, subsets defined based on area) in Fig. 3f. In addition to the general tendency of decreasing averaged L-moment ratios with increasing area and MAP values, an interesting feature is observed in the L-Cv versus area diagram in Fig. 3a. If one looks at the single stations (grey dots) there is an ascending-descending relationship of the $\mathrm{L}-\mathrm{Cv}$ values with increasing area, with a maximum located around $100 \mathrm{~km}^{2}$. This was also found, among others, by Blöschl and Sivapalan (1997) and Iacobellis et al. (2002) in more regional contexts, and it is relevant to find it as well in the present study. 

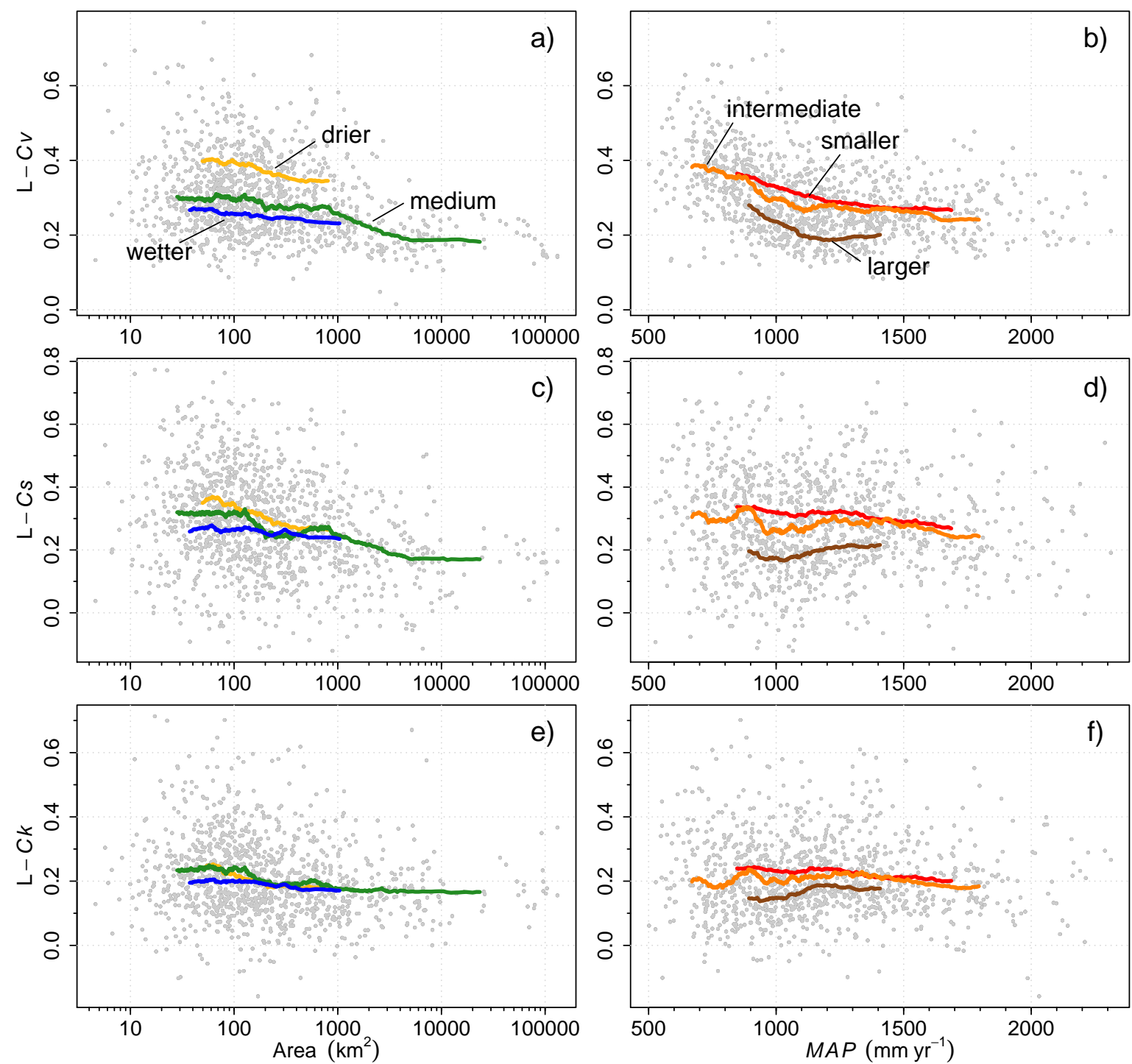

Figure 3. Sample L-Cv, L-Cs and L-Ck values for each catchment (grey points) plotted against catchment area and MAP. Lines show the record length WMAs over 70 catchments for the subsets smaller, intermediate, larger, drier, medium, and wetter defined in Fig. 2.

\subsection{Area and MAP control on regional flood frequency distribution}

Acknowledging the influence of both catchment size and mean annual precipitation on the L-moment ratios, the next step in the analysis is to assess the impact of this influence on the underlying regional parent distribution of annual flood sequences. This investigation is based on the novel use of Lmoment ratio diagrams, which, in this context, are used to analyse the sensitivity of the choice of a parent distribution to the catchment and climate characteristics. The two types of L-moment ratio diagrams mentioned in Sect. 2 are used, namely (i) L-Cv-L-Cs and (ii) L-Cs-L-Ck. The former is used in this study for assessing the suitability of the commonly used two-parameter distributions of Gumbel (GUM), gamma (GAM), two-parameter lognormal (LN2) and exponential (EXP). The latter is used in connection with the threeparameter distributions generalized logistic (GLO), generalized extreme value (GEV), three-parameter log-normal (LN3) and Pearson type 3 (PE3). These distributions, presented in the companion paper by Salinas et al. (2014), were found to be preferentially recommended in national guidelines for flood frequency estimation from several European countries (Castellarin et al., 2012). The assessment of which statistical model fits better the averaged statistical properties of the sample was done visually based on the distance between the averaged sample L-moment ratios and the theoretical lines, as objective goodness of fit measures require the flood peak data (Laio, 2004) and, in this case, only the Lmoment ratios were available. 

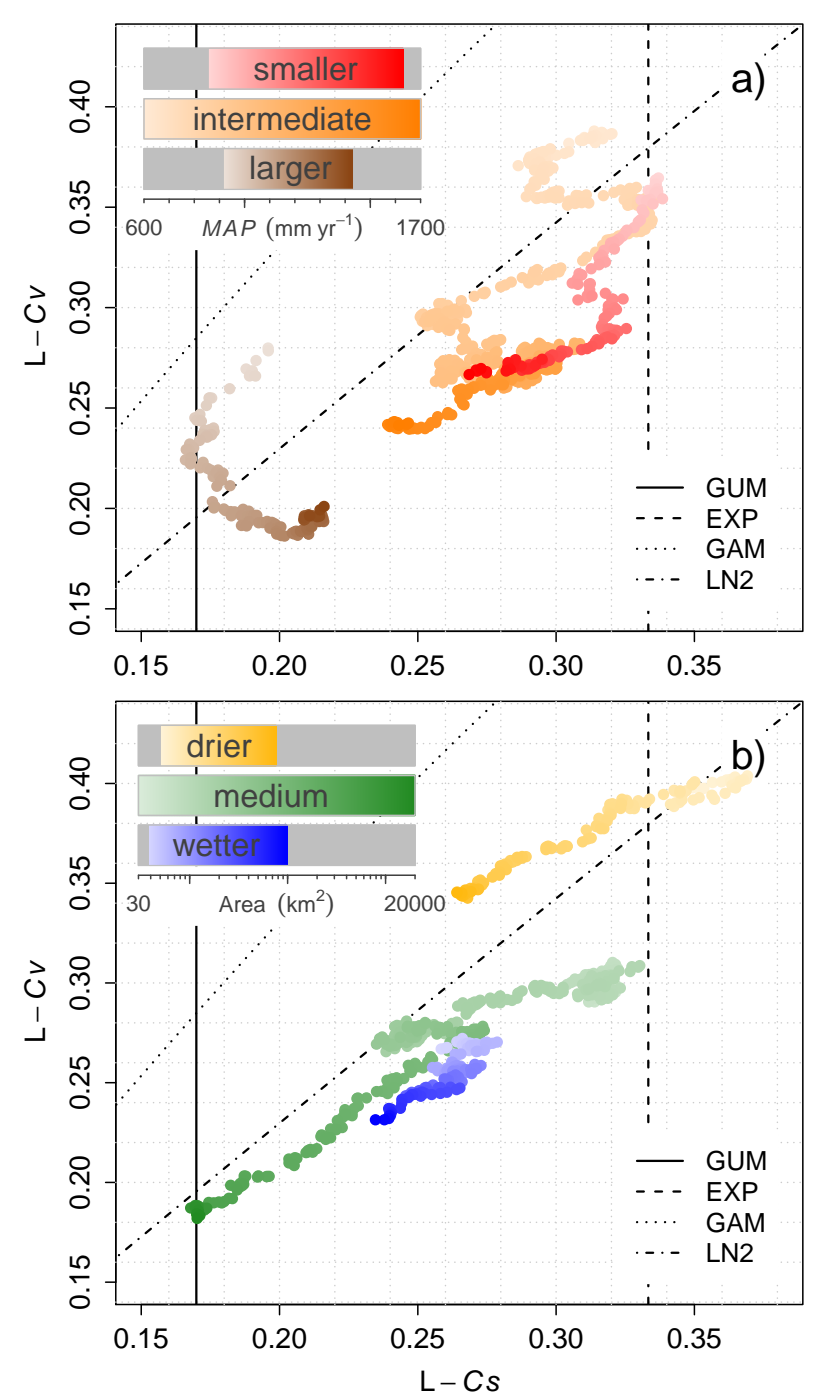

Figure 4. L-moment ratio diagrams for the subsets defined by (a) catchment area (smaller, intermediate, larger), and (b) MAP (wetter, medium, drier) described in Fig. 3. Each point represents the record length WMA over 70 catchments of L-Cv against corresponding values of L-Cs and the colour intensity is proportional to (a) MAP and (b) catchment area.

\subsubsection{Two-parameter distributions}

The diagrams in Fig. 4 report the WMA values of L-Cs and $\mathrm{L}-\mathrm{Cv}$ associated with a given average value of catchment area or MAP for the same moving windows for the 70 catchments defined in the previous section and shown in Fig. 3, again stratified in smaller, intermediate and larger catchments (Fig. 4a) and wetter, medium and drier catchments (Fig. 4b). To emphasize the influence of the catchment descriptors (i.e. area or MAP), the colour intensity of each plotted WMA value has been graded according to the value of the catchment descriptor that has not been used for the stratification, with increasing intensity for increasing de- scriptor value. For example, Fig. 4a shows the WMA values when the data set is divided by catchment size into smaller, intermediate and larger catchments, and the colour grading of the points reflects the mean value of MAP for each subset of the 70 catchments. More precisely, red WMA values of L-Cv and L-Cs in Fig. 4a correspond to the transect associated with smaller basins in the catchment descriptor space defined by MAP, orange relates to the intermediate-sizedbasins transect in the catchment descriptor space defined by MAP and brown points stand for the larger-basins transect in the catchment descriptor space defined by MAP. Analogously, in Fig. 4b, yellow WMA values of L-Cv and L-Cs represent the transect associated with the drier basins in the catchment descriptor space defined by catchment area, green corresponds to the medium MAP basins transect in the catchment descriptor space defined by area, and blue points relate to the wetter basins transect in the catchment descriptor space defined by area.

The position of the WMA values of samples $\mathrm{L}-\mathrm{Cv}$ and $\mathrm{L}$ Cs relative to the theoretical distributions shown in Figs. 4a and $b$ indicate that none of the considered two-parameter distributions fits the statistical properties of the data sets. In particular, both figures show that the WMA values of sample L-Cs are always larger than that of the Gumbel distribution (fixed value of 0.1699) and smaller than that of the exponential distribution (fixed value of 0.3333), with the exception of values up to 0.37 for the smallest drier (low MAP) catchments (less intense yellow points in Fig. 4b). Sample values of L-moment ratios do not seem to follow systematically the shape of any of the lines representing the theoretical L-Cv and L-Cs relationships of the considered twoparameter distributions, but some WMA values tend to lie closer to the LN2 curve than to any other. This is the case for the intermediate-sized and medium MAP catchments of the data set (midintensity orange and midintensity green points in Figs. 4a and b, respectively). The only subset for which sample values approach the statistical properties of the Gumbel distribution, and they do it towards the intersection with the LN2 curve, is for larger and medium MAP catchments (intense green points in Fig. 4b and, to some extent, midintensity brown points in Fig. 4a). The subset corresponding to drier catchments presents the largest $\mathrm{L}-\mathrm{Cv}$ and $\mathrm{L}-\mathrm{Cs}$ values, while the smallest L-moment ratios are found for the subset of larger catchments lying as mentioned before closer to the Gumbel line than the rest of WMA values. Inside each subset (i.e. smaller, intermediate, larger, drier, medium or wetter basins) the intensity of gradation increases with decreasing $\mathrm{L}-\mathrm{Cv}$ and $\mathrm{L}-\mathrm{Cs}$ values. This means that for larger values of catchment area and MAP, lower regionally averaged values of L-Cv and L-Cs are expected. The gradients are clearer for the smaller and drier catchments (red and yellow points in Fig. 4), while there is a slight increase of the WMA LCs values for wetter catchments inside the larger catchments subset. This could be attributed to the fact that, as pointed out in Sect. 2, all larger catchments have a similar averaged 
MAP value (between $1000-1400 \mathrm{~mm} \mathrm{yr}^{-1}$ ) belonging to the same intermediate wetness subset and the differences in the rainfall regime are not big enough to draw conclusions about their control on the averaged L-Cs values inside the group.

\subsubsection{Three-parameter distributions}

Figures 5 and 6 report the L-moment diagrams defined by plotting WMA values of L-Cs and L-Ck in a similar fashion to Fig. 4. In this case, the two intermediate subsets (i.e intermediate-sized and medium MAP values) were plotted separately from the subsets defining smaller, larger, drier and wetter catchments to ease the visual interpretation of the plots (as far as possible avoiding overlapping points). Figure 5a shows the subset of WMA values derived for the smaller and larger catchments, with the colour intensity representing the average MAP value and Fig. 5b illustrates intermediate-sized catchments with the gradation representing again the average MAP value. Similarly, Fig. 6a shows the subset of WMA values representing drier and wetter catchments, with the colour intensity representing catchment size, while Fig. $6 \mathrm{~b}$ is relative to the subset characterized by intermediate MAP values.

Figure 5a shows that the WMA values associated with larger catchments are located closer to the GEV line than to any other distribution, generally showing slightly higher L$\mathrm{Ck}$ values than expected for a GEV distribution. For smaller catchments, the GEV is the distribution that best represents the statistical properties of the sample, being the scatter of points much closer to the theoretical curve than that from the subset of larger catchments. For intermediate-sized catchments, Fig. 5b highlights a strong control of MAP on the appropriate distribution; medium-sized catchments associated with high MAP values are situated closer the curve of the GEV distribution, while catchments with lower MAP values move towards the LN3 distribution. This implies that, for drier catchments inside the intermediate-sized subset, the distribution type shifts to a more skewed one (for the same L-Ck, the LN3 has higher L-Cs values than GEV).

Figure 6a shows the WMA values for the two subsets including the drier and wetter catchments as defined by the MAP values. The WMA values associated with wetter catchments are located closer to the line defining the GEV distribution, suggesting that GEV is an appropriate distribution for wetter catchments more or less regardless of catchment size (as determined by the blue colour gradation). In contrast, the statistical properties of the drier catchments are better represented by the LN3 distribution, as also illustrated in Fig. 5b. Catchments with intermediate MAP values are associated with a larger range of L-Cs values depending on their size (see Fig. 6b), and lie closer to the GEV line than to any other distribution considered, with a slight tendency for the smallest and largest catchments inside the subset to exhibit higher values of L-Ck than expected from a GEV distribution.
Analogously to Fig. 4, the area and MAP control on the position of the relative WMA values between the subsets remain in Figs. 5 and 6, showing again higher averaged Lmoment ratio values when comparing smaller to larger catchments and drier to wetter catchments.

\section{Discussion}

Previous sections have highlighted the importance of linking the flood generation processes to the observed L-moment ratios of the annual maxima sequences, and the position of the regional averages at the diagrams, in order to understand from the differences between catchments in terms of underlying parent distributions. Two lumped-catchment descriptors are used as surrogate covariates representing the spatially distributed and complex hydrological processes controlling the catchment flood response. Precisely, the area of the basin is an indicator of the scale interplays between catchment processes and rainfall (Blöschl and Sivapalan, 1995), while mean annual precipitation acts as control of probabilistic behaviour of floods through its effect on antecedent soil moisture conditions (Sivapalan et al., 2005), and also provides an indication about other local and atmospheric process.

For example, low MAP values could indicate regions with prevalence for more localized and variable storms, usually flashier in time and with higher rainfall intensities. This higher between-years variability and skewed distribution of rainfall extreme intensities translates into higher $\mathrm{L}-\mathrm{Cv}$ and L-Cs values of annual floods, as Figs. $4 \mathrm{~b}$ and $6 \mathrm{a}$ suggest. In contrast, long-duration frontal or advective events, associated with larger spatial extensions and lower rainfall intensities, are expected at catchments presenting higher MAP values, more clearly shown in Fig. 6a. These two kinds of precipitation regimes will also have an effect on the co-evolution of landform with hydrological processes (Gaál et al., 2012), in which rainfall plays an important role at multiple timescales. The variability of flood magnitude between years, and the Lcoefficient of variation as a measure of this variability, tends to be higher in smaller and intermediate-sized catchments, compared to the larger ones, as shown in Fig. 4. The main reasons are both the spatial heterogeneity of rainfall and the interaction between the spatial and temporal scales of rainfall and catchment size taking place. This interplay causes the catchment to resonate with storms of similar spatio-temporal extension. In the case of smaller basins this corresponds to short duration, high intensity, spatially concentrated storms (i.e. convective events or flash floods), which are also typical of drier climates; while in larger catchments the resonance appears with longer storms, usually associated with lower intensities, with a bigger spatial extension (i.e. advective or frontal events), more typical of wetter environments (see e.g. Blöschl and Sivapalan, 1995; Sivapalan and Blöschl, 1998). These two differentiated regimes for rainfall extremes will 

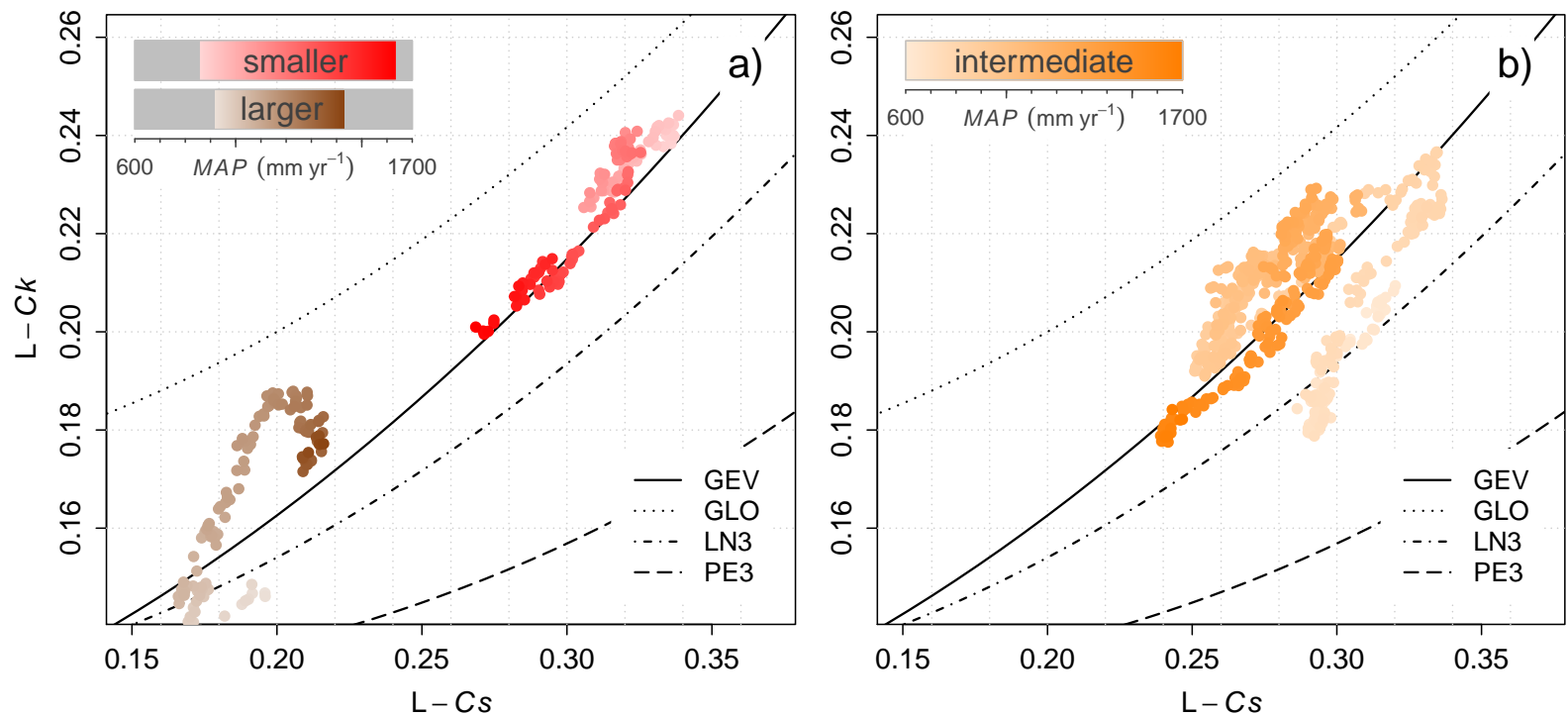

Figure 5. L-moment ratio diagrams for the subsets defined by catchment area: (a) smaller, larger, and (b) intermediate described in Fig. 3. Each point represents the record length WMA over 70 catchments of L-Ck against corresponding values of L-Cs and the colour intensity is proportional to MAP.
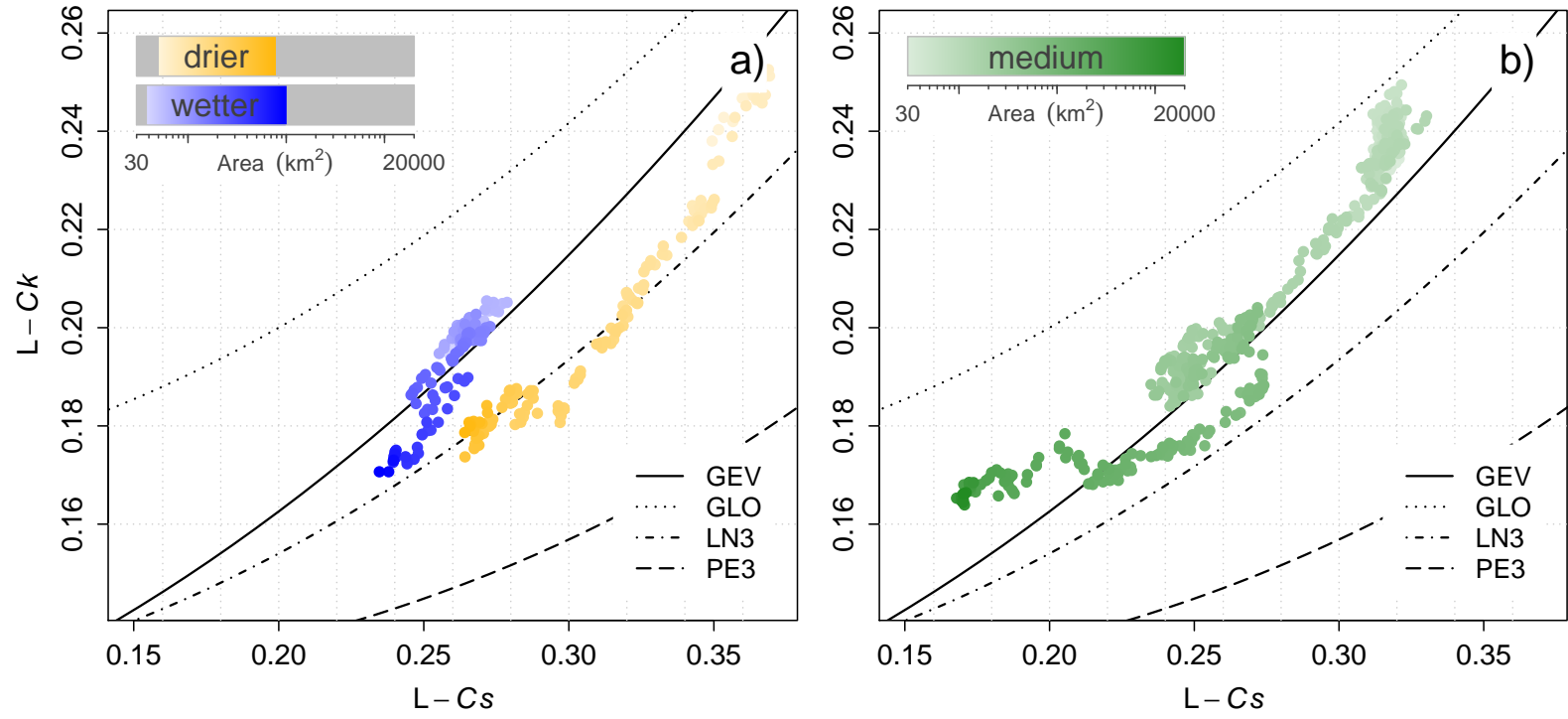

Figure 6. L-moment ratio diagrams for the subsets defined by MAP: (a) drier, wetter, and (b) medium described in Fig. 3. Each point represents the record length WMA over 70 catchments of L-Ck against corresponding values of L-Cs and the colour intensity is proportional to catchment area.

cause not only a higher L-coefficient of variation but also a higher L-coefficient of skewness in the flood distributions for smaller and drier catchments compared to the larger and wetter ones, as shown in Fig. 5a. Aside from precipitation input, other catchment processes can also play an important role in shaping the properties of the flood distribution. The presence of non-linearities in runoff production and routing in smaller, drier basins (Medici et al., 2008) in contrast to the aggregation of processes in larger catchments (Sivapalan et al., 2002) will translate in decreasing values of L-Cv and
L-Cs with increasing values of MAP and, more strongly, catchment size. One visible consequence of the higher dispersion and skewness of the flood frequency distributions with decreasing catchment area and increasing aridity is the fact that predicting flood magnitudes and exceedance probabilities in ungauged basins is more difficult in smaller, more arid catchments, as compared to bigger, less arid ones (see e.g. Salinas et al., 2013).

Therefore, the main findings from the analysis presented in the previous sections need to be interpreted in a hydrological 
way, instead of in a merely statistical sense. For example, the fact that the GEV distribution is found to be the model representing better the averaged statistical properties of catchments with medium to high values of MAP regardless of size, is probably because few of the catchments in arid regions with highly skewed distributions of rainfall extremes are present in these subclasses. In contrast, there is a clear indication that the LN3 distribution, which has a higher skewness than GEV for a given kurtosis, reproduces better the sample properties of the drier, intermediate-sized subset, representing most likely other flood generation processes than for the data subsets more affine to the GEV distribution. Nevertheless, the limited number of catchments classified simultaneously as smaller and drier, larger and wetter, or larger and drier prevents these conclusions from being extended further.

The LN2 distribution represents in some circumstances a valid alternative to the other commonly used threeparameter distributions, especially for intermediate-sized, medium MAP catchments. The fact of having one parameter less than the GEV or the LN3 allows the LN2 to reproduce only a limited range of hydrological processes maybe not able to capture the extreme cases of the smaller or drier catchments. Sample L-Cs values are shown to be, on a regional average, higher than the ones of the Gumbel distribution, being the larger and medium MAP catchments the ones closer to its theoretical curve. This is likely due to the fixed skewness value of the Gumbel distribution, relatively low for the regional averages of the given data set and the selected aggregation levels, corresponding substantially to the smoother processes in larger catchments. Also, for the smaller, wetter, and drier catchment subclasses, none of the considered two-parameter distributions is capable of accurately representing the averaged values of the subset.

Recalling the scientific questions presented in the introduction, this study has been able to quantify the controls of area and mean annual precipitation on the sample L-moment ratios of annual maximum flood discharges, and has shown how these controls may guide the selection of suitable parent distributions in an L-moments diagram framework. Additionally, the novel use of traditional L-moment ratio diagrams presented in Figs. 4, 5 and 6 may be very informative, and could help to better understand the changes in flood hazard resulting from different sources of environmental change. By explicitly accounting for the conceptual process controls through catchment descriptors (catchment area and MAP in this study), the sensitivity of the flood frequency distribution to changes in process controls can be determined. For example, Figs. 5b and 6a show that for medium-sized catchments the most appropriate distribution changes from a GEV to a LN3 distribution as MAP decreases. Thus, if future climate projections indicate a reduction of MAP, then the results in Figs. 5b and 6a suggest that the corresponding change in flood distribution is likely to be characterized by a move towards a larger skewness (e.g. from a GEV to a LN3 distribution), assuming that the current relationship between MAP and storm rainfall intensity distributions holds in future climates. This sensitivity analysis could be extended by including additional catchment descriptors representing processes likely to change, e.g. land cover and urbanization, and by weighting each distribution type in case a multimodel approach is selected for representing the regional flood frequency distribution (see e.g. Laio et al., 2009).

\section{Conclusions}

This study has shown that the inclusion of information on the underlying hydrological processes in the model choice is of high importance. Each catchment has been characterized in terms of size and mean annual precipitation, as these properties have previously been found to be rough surrogates for the different flood generation processes, but also because a survey presented in the companion paper by Salinas et al. (2014) showed that only these, the most elemental catchment properties, are readily available across Europe. Some preliminary conclusions can be drawn, such as the shift from a GEV to a LN3 as a more appropriate distribution for decreasing MAP values in intermediate-sized catchments. However, the robustness of these statements is limited due to the lack of more data from simultaneous extremes of the subclasses (e.g. smaller and drier catchments) but maybe more due to the absence of better catchment attributes that allow us to fully describe the flood generation processes.

Several studies of flood hydrology have also highlighted the potential utility of soil and land-use data for characterizing flood frequency curves in ungauged European catchments. Thus, there are potentially larger benefits associated with future development of consistent pan-European catchment descriptor data sets as a fundamental step in harmonizing methods. In particular for this database, there is a variety of other catchment and climatic descriptors that could potentially improve the analysis. As pointed out in Sect. 2, there is a large number of catchments that could be strongly affected by snow processes. Its control on the flood regimes could be analysed through surrogates such as median (or maximum) catchment elevation, mean annual air temperature or by more specific ones like the fraction of solid to liquid precipitation. Also, having information on a station basis about the dominant event types and the precipitation that most likely triggers the hydrological extremes (convective rainfall, long rain events, etc.) might allow us to have a better understanding of the whole hydrological regime which could be used to more accurately sort the database. For example, a recent study by Gaál et al. (2014) suggested the use of lightning data as an indicator of convectivity in rainfall events. Additionally, there are other climatic indicators related to the energy and water balance, such as the aridity index (ratio between potential evapotranspiration and mean annual precipitation), that could provide together with rainfall and temperature valuable information on the climate type. 
The original utilization of the traditional L-moment ratio diagrams presented in this study, in conjunction with a more refined characterization of European catchments based upon a richer catchment descriptor data set, could also contribute to better understanding the modifications in flood hazard resulting from different sources of environmental change, and to move further towards the definition of a set of "processdriven" pan-European flood frequency distributions.

Acknowledgements. The authors are grateful for the financial contribution made by the COST Office grant ES0901 European procedures for flood frequency estimation (FloodFreq) and for contributions made by members of Working Group 2 of the COST Action. Funding from the ERC FloodChange project (ERC Advanced Grant FP7-IDEAS-ERC-AG-PE10 291152) and from the Austrian Science Funds (FWF) as part of the Vienna Doctoral Programme on Water Resource Systems (DK-plus W1219-N22) is also acknowledged. The compilation of the Italian data set was partially supported by the Italian Government through its national grant to the programme on Characterisation of Ungauged Basins by Integrated uSe of hydrological Techniques (CUBIST Project) and the compilation of the Slovakian data set was partially supported by the Agency for Research and Development under contract no. APVV-0303-11. Both supports are greatly acknowledged. Referees F. Laio, R. Romanowicz, S. Grimaldi, and V. Iacobellis, as well as Günter Blöschl, are thankfully acknowledged for their constructive comments and suggestions. Juraj Parajka is greatly acknowledged for preparing the map in Fig. 1.

Edited by: R. Woods

\section{References}

Blöschl, G. and Sivapalan, M.: Scale issues in hydrological modelling: A review, Hydrol. Process., 9, 251-290, doi:10.1002/hyp.3360090305, 1995.

Blöschl, G. and Sivapalan, M.: Process controls on regional flood frequency: coefficient of variation and basin scale, Water Resour. Res., 33, 2967-2980, doi:10.1029/97WR00568, 1997.

Bogdanowicz, E.: Multimodel approach to estimation of Extreme Value distribution quantiles (in Polish), Hydrology in Eng. and Water Management (Hydrologia w Inżynierii i Gospodarce Wodnej), edited by: Wiezik, B., Polish Academy of Sciences, Environmental Eng. Committee, Monographs, 68, 57-70, 2010.

Brath, A., Castellarin, A., and Montanari, A.: Assessing the reliability of regional depth-duration-frequency equations for gaged and ungaged sites, Water Resour. Res., 39, 1367, doi:10.1029/2003WR002399, 2003.

Castellarin, A., Kohnová, S., Gaál, L., Fleig, A., Salinas, J. L., Toumazis, A., Kjeldsen, T. R., and Macdonald, N.: Review of applied statistical methods for flood frequency analysis in Europe, Milestone Report for WG2 of COST Action ES0901, Tech. rep., Centre for Ecology \& Hydrology, Wallingford, UK, on behalf of COST, available at: (http://www.cost.eu/media/publications/), 2012.
Di Baldassarre, G., Castellarin, A., and Brath, A.: Relationships between statistics of rainfall extremes and mean annual precipitation: an application for design-storm estimation in northern central Italy, Hydrol. Earth Syst. Sci., 10, 589-601, doi:10.5194/hess-10-589-2006, 2006.

El Adlouni, S., Bobée, B., and Ouarda, T. B. M. J.: On the tails of extreme event distributions in hydrology, J. Hydrol., 355, 1-4, doi:10.1016/j.jhydrol.2008.02.011, 2008.

Farquharson, F. A. K., Meigh, J. R., and Sutcliffe, J. V.: Regional flood frequency analysis in arid and semi-arid areas, J. Hydrol., 138, 487-501, 1992.

Gaál, L., Szolgay, J., Kohnová, S., Parajka, J., Merz, R., Viglione, A., and Blöschl, G.: Flood timescales: understanding the interplay of climate and catchment processes through comparative hydrology, Water Resour. Res., 48, W04511, doi:10.1029/2011WR011509, 2012.

Gaál, L., Molnar, P., and Szolgay, J.: Selection of intense rainfall events based on intensity thresholds and lightning data in Switzerland, Hydrol. Earth Syst. Sci., 18, 1561-1573, doi:10.5194/hess-18-1561-2014, 2014.

Hosking, J. R. M. and Wallis, J. R.: Regional Frequency Analysis: an Approach Based on L-Moments, Cambridge University Press, Cambridge, UK, 1997.

Iacobellis, V., Claps, P., and Fiorentino, M.: Climatic control on the variability of flood distribution, Hydrol. Earth Syst. Sci., 6, 229 238, doi:10.5194/hess-6-229-2002, 2002.

Kjeldsen, T. R.: COST Action ES0901: European procedures for flood frequency estimation (FloodFreq), Geophys. Res. Abs., 13, EGU2011-10327, 2011.

Kochanek, K., Strupczewski, W. G., and Bogdanowicz, E.: On seasonal approach to flood frequency modelling. Part II: flood frequency analysis of Polish rivers, Hydrol. Process., 26, 717-730, doi:10.1002/hyp.8178, 2012.

Laio, F.: Cramer-von Mises and Anderson-Darling goodness of fit tests for extreme value distributions with unknown parameters, Water Resour. Res., 40, W09308, doi:10.1029/2004WR003204, 2004.

Laio, F., Di Baldassarre, G., and Montanari, A.: Model selection techniques for the frequency analysis of hydrological extremes, Water Resour. Res., 45, W07416, doi:10.1029/2007WR006666, 2009.

Medici, C., Butturini, A., Bernal, S., Vázquez, E., Sabater, F., Vélez, J. I., and Francés, F.: Modelling the non-linear hydrological behaviour of a small Mediterranean forested catchment, Hydrol Process., 22, 3814-3828, doi:10.1002/hyp.6991, 2008.

Meigh, J. R., Farquharson, F. A. K., and Sutcliffe, J. V.: A worldwide comparison of regional flood estimation methods and climate, Hydrol. Sci. J., 42, 225-244, doi:10.1080/02626669709492022, 1997.

Merz, R. and Blöschl, G.: A process typology of regional floods, Water Resour. Res., 39, 1340, doi:10.1029/2002WR001952, 2003.

Merz, R. and Blöschl, G.: Process controls on the statistical flood moments - a data based analysis, Hydrol. Process., 23, 675-696, doi:10.1002/hyp.7168, 2009.

Padi, P. T., Di Baldassarre, G., and Castellarin, A.: Floodplain management in Africa: Large scale analysis of flood data, Phys Chem. Earth, 36, 292-298, doi:10.1016/j.pce.2011.02.002, 2011. 
Peel, M. C., Wang, Q. J., Vogel, R. M., and McMahon, T. A.: The utility of L-moment ratio diagrams for selecting a regional probability distribution, Hydrol. Sci. J., 46, 147-156, doi:10.1080/02626660109492806, 2001.

Robson, A. J. and Reed, D. W.: Flood Estimation Handbook (FEH), chap. Statistical procedures for flood frequency estimation, p. Vol. 3, Institute of Hydrology, Wallingford, UK, 1999.

Salinas, J. L., Laaha, G., Rogger, M., Parajka, J., Viglione, A., Sivapalan, M., and Blöschl, G.: Comparative assessment of predictions in ungauged basins - Part 2: Flood and low flow studies, Hydrol. Earth Syst. Sci., 17, 2637-2652, doi:10.5194/hess-172637-2013, 2013.

Salinas, J. L., Castellarin, A., Viglione, A., Kohnová, S., and Kjeldsen, T. R.: Regional parent flood frequency distributions in Europe - Part 1: Is the GEV model suitable as a pan-European parent?, Hydrol. Earth Syst. Sci., 18, 4381-4389, doi:10.5194/hess18-4381-2014, 2014.

Schaefer, M. G.: Regional analyses of precipitation annual maxima in Washington State, Water Resour. Res., 26, 119-131, doi:10.1029/WR026i001p00119, 1990.

Sivapalan, M. and Blöschl, G.: Transformation of point rainfall to areal rainfall: intensity-duration-frequency curves, J. Hydrol., 204, 150-167, doi:10.1016/S0022-1694(97)00117-0, 1998.

Sivapalan, M., Jothityangkoon, C., and Menabde, M.: Linearity and nonlinearity of basin response as a function of scale: discussion of alternative definitions, Water Resour. Res., 38, 4-1-4-5, doi:10.1029/2001WR000482, 2002.
Sivapalan, M., Blöschl, G., Merz, R., and Gutknecht, D.: Linking flood frequency to longterm water balance: Incorporating effects of seasonality, Water Resour. Res., 41, W06012, doi:10.1029/2004WR003439, 2005.

Thompson, E. M., Baise, L. G., and Vogel, R. M.: A global index earthquake approach to probabilistic assessment of extremes, J. Geophys. Res., 112, B06314, doi:10.1029/2006JB004543, 2007.

Viglione, A.: Confidence intervals for the coefficient of L-variation in hydrological applications, Hydrol. Earth Syst. Sci., 14, 2229 2242, doi:10.5194/hess-14-2229-2010, 2010.

Viglione, A., Castellarin, A., Rogger, M., Merz, R., and Blöschl, G.: Extreme rainstorms: comparing regional envelope curves to stochastically generated events, Water Resour. Res., 48, W01509, doi:10.1029/2011WR010515, 2012.

Vogel, R. M. and Fennessey, N.: L moment diagrams should replace product moment diagrams, Water Resour. Res., 29, 1745-1752, doi:10.1029/93WR00341, 1993.

Vogel, R. M. and Wilson, I.: The probability distribution of annual maximum, minimum and average streamflow in the United States, J. Hydrol. Eng., 1, 69-76, doi:10.1061/(ASCE)10840699(1996)1:2(69), 1996. 